\title{
Pilot randomised trial of a healthy eating behavioural intervention in uncontrolled asthma
}

\author{
Jun $\mathrm{Ma}^{1,2,3}$, Peg Strub ${ }^{4}, \mathrm{Nan}_{\mathrm{Lv}}{ }^{1}$, Lan Xiao ${ }^{1}$, Carlos A. Camargo Jr ${ }^{5}$, \\ A. Sonia Buist ${ }^{6}$, Philip W. Lavori ${ }^{7}$, Sandra R. Wilson ${ }^{1,2}$, Kari C. Nadeau ${ }^{8}$ and \\ Lisa G. Rosas ${ }^{1,2}$
}

\begin{abstract}
Affiliations: ${ }^{1}$ Palo Alto Medical Foundation Research Institute, Palo Alto, CA, USA. ${ }^{2}$ Dept of Medicine, Stanford University School of Medicine, Stanford, CA, USA. ${ }^{3}$ Dept of Health Policy and Administration, University of Illinois at Chicago School of Public Health, Chicago, IL, USA. "Dept of Allergy, Asthma, and Immunology, The Permanente Medical Group, San Francisco Medical Center, San Francisco, CA, USA. ${ }^{5}$ Dept of Emergency Medicine, Massachusetts General Hospital, Harvard Medical School, Boston, MA, USA. ${ }^{6}$ Pulmonary \& Critical Care Medicine, Oregon Health \& Science University, Portland, OR, USA. ${ }^{7}$ Dept of Health Research \& Policy, Stanford University School of Medicine, Stanford, CA, USA. ${ }^{8}$ Dept of Paediatric Allergy and Immunology, Stanford University, Stanford, CA, USA.
\end{abstract}

Correspondence: Jun Ma, Dept of Health Policy and Administration, School of Public Health, University of Illinois at Chicago, Chicago, IL 60607, USA. E-mail: maj2015quic.edu

ABSTRACT Rigorous research on the benefit of healthy eating patterns for asthma control is lacking.

We randomised 90 adults with objectively confirmed uncontrolled asthma and a low-quality diet (Dietary Approaches to Stop Hypertension (DASH) scores $<6$ out of 9) to a 6 -month DASH behavioural intervention $(n=46)$ or usual-care control $(n=44)$. Intention-to-treat analyses used repeated-measures mixed models.

Participants were middle-aged, $67 \%$ female and multiethnic. Compared with controls, intervention participants improved on DASH scores (mean change $(95 \% \mathrm{CI}) 0.6(0,1.1)$ versus $-0.3(-0.8,0.2)$; difference $0.8(0.2,1.5))$ and the primary outcome, Asthma Control Questionnaire scores $(-0.2(-0.5,0)$ versus $0(-0.3,0.3)$; difference $-0.2(-0.5,0.1))$ at 6 months. The mean group differences in changes in Mini Asthma Quality of Life Questionnaire overall and subdomain scores consistently favoured the intervention over the control group: overall 0.4 (95\% CI $0,0.8)$, symptoms $0.5(0,0.9)$, environment 0.4 $(-0.1,1.0)$, emotions $0.4(-0.2,0.9)$ and activities $0.3(0,0.7)$. These differences were modest, but potentially clinical significant.

The DASH behavioural intervention improved diet quality with promising clinical benefits for better asthma control and functional status among adults with uncontrolled asthma. A full-scale efficacy trial is warranted.

@ERSpublications

A dietary programme has clinical benefits for asthma control and functional status in adults with uncontrolled asthma http://ow.ly/R8zUn

This article has supplementary material available from erj.ersjournals.com

Received: April 142015 | Accepted after revision: July 162015 | First published online: Oct 222015

Clinical trial: This study is registered at ClinicalTrials.gov with identifier number NCT01725945.

Support statement: This research was supported by grant R34 HL108753 from the National Heart, Lung and Blood Institute, and internal funding from the Palo Alto Medical Foundation Research Institute. P.W. Lavori acknowledges support by the Clinical and Translational Science Award 1UL1 RR025744 for the Stanford Centre for Clinical and Translational Education and Research (Spectrum) from the National Centre for Research Resources. The content is solely the responsibility of the authors and does not necessarily represent the official views of the National Heart, Lung and Blood Institute. No sponsor or funding source had a role in the design or conduct of the study; collection, management, analysis or interpretation of the data, or preparation, review or approval of the manuscript. Funding information for this article has been deposited with FundRef.

Conflict of interest: Disclosures can be found alongside the online version of this article at erj.ersjournals.com

Copyright OERS 2016 


\section{Introduction}

Asthma prevalence has increased in recent years, currently affecting $>18$ million US adults [1]. Diet composition changes and worsened diet quality have been implicated as contributing factors in this trend [2], as well as poor asthma control [3]. Some studies have investigated the potential benefit of certain foods (e.g. fruit, vegetables and fish) and nutrients (e.g. vitamins C, D and E, and $\omega-3$ fatty acids) for improving asthma control in adults; however, the evidence is mixed and of insufficient quality to inform clinical and public health practice [4-7]. Several more recent studies focussed on dietary patterns rather than specific foods or nutrients and their association with asthma control in adults [6, 8]. A recent meta-analysis by Lv et al. [9] showed no association between dietary patterns and asthma prevalence or incidence in adults, although some individual studies included in the systematic review reported significant associations between dietary patterns and risk of uncontrolled asthma, measures of lung function such as forced expiratory volume in $1 \mathrm{~s}$ (FEV1) and/or frequency of asthma attacks. The review identified only one published randomised controlled trial (RCT) that examined two Mediterranean diet interventions compared with no-intervention control in 38 adults with symptomatic asthma. The trial showed the feasibility of improving Mediterranean diet scores, but no beneficial effect of either intervention on asthma control, lung function, asthma-related quality of life or inflammatory markers [8]. Additional RCTs are needed to examine the efficacy of healthy dietary patterns for asthma control.

In this RCT pilot study, "DASH for Asthma", we sought to examine the potential efficacy of a behavioural intervention promoting Dietary Approaches to Stop Hypertension (DASH) to improve asthma control in adults with uncontrolled asthma and to determine whether a full-scale trial would be warranted according to a priori decision rules. The main study aims were to estimate the $95 \%$ confidence intervals of the net-of-control effects of the DASH behavioural intervention on the primary outcome, change in asthma control measured by the Juniper Asthma Control Questionnaire (ACQ) [10], and on secondary outcomes including specific manifestations of asthma indicative of current impairment (e.g. lung function, symptoms and rescue medication use) and future risk (e.g. asthma exacerbations). In addition, changes in DASH concordance index, adherence to the primary DASH daily goals, and changes in weight, blood pressure and lipids were assessed.

\section{Methods}

An Institutional Review Board of the Kaiser Foundation Research Institute in Northern California and the study Data and Safety Monitoring Board (DSMB) approved the study. All participants provided written informed consent. The trial protocol was published previously [11].

\section{Study participants}

Participants were recruited (February 15-December 12, 2013) from the Kaiser Permanente medical centres in San Francisco and Hayward. Inclusion criteria included age 18-70 years, body mass index (BMI) $18.5-39.9 \mathrm{~kg} \cdot \mathrm{m}^{-2}$, consuming a low-quality diet (defined as score $<6$ out of 9 total on the DASH concordance index summing nine nutrient targets, including total fat, saturated fat, protein, cholesterol, fibre, magnesium, calcium, sodium and potassium [12]) and confirmation of uncontrolled persistent asthma through a multistage screening process (i.e. electronic asthma registry queries, completion of the Asthma Control Test [13], pre- and post-bronchodilator spirometry, and if necessary, medical chart review by an asthma specialist) (online supplementary table E1). Exclusion criteria included serious medical or psychiatric conditions (e.g. chronic obstructive pulmonary disease, stroke, psychosis) or special life circumstances (e.g. pregnancy, planned relocation). Of the 2241 patients mailed recruitment letters after their primary care provider approved study contact, 805 patients declined participation, 757 were ineligible and 589 were not contactable or nonresponsive. This process yielded the target enrolment of 90 eligible and consenting participants (figure 1).

\section{Randomisation and blinding}

Participants were randomly assigned in a 1:1 ratio to receive the DASH intervention $(\mathrm{n}=46)$ or no intervention $(\mathrm{n}=44)$ while all participants continued usual care. Using a Web-based random allocation system [14], we applied Pocock's minimisation, a covariate-adaptive randomisation method [15], to achieve better-than-chance between-treatment balance across six prognostic factors (age, sex, race/ ethnicity, smoking status, DASH concordance index and the seven-item ACQ score). Efron's biased-coin method [16] was employed to protect allocation concealment with use of non-extreme randomisation probabilities (i.e. $2 / 3$ to $1 / 3$ ). A designated study staff person who did not have the ability to influence the allocation system's execution performed randomisation. The trial design precluded blinding participants or interventionists to treatment assignment; however, the investigators, DSMB members, outcome assessors and data analyst were masked throughout the trial. 


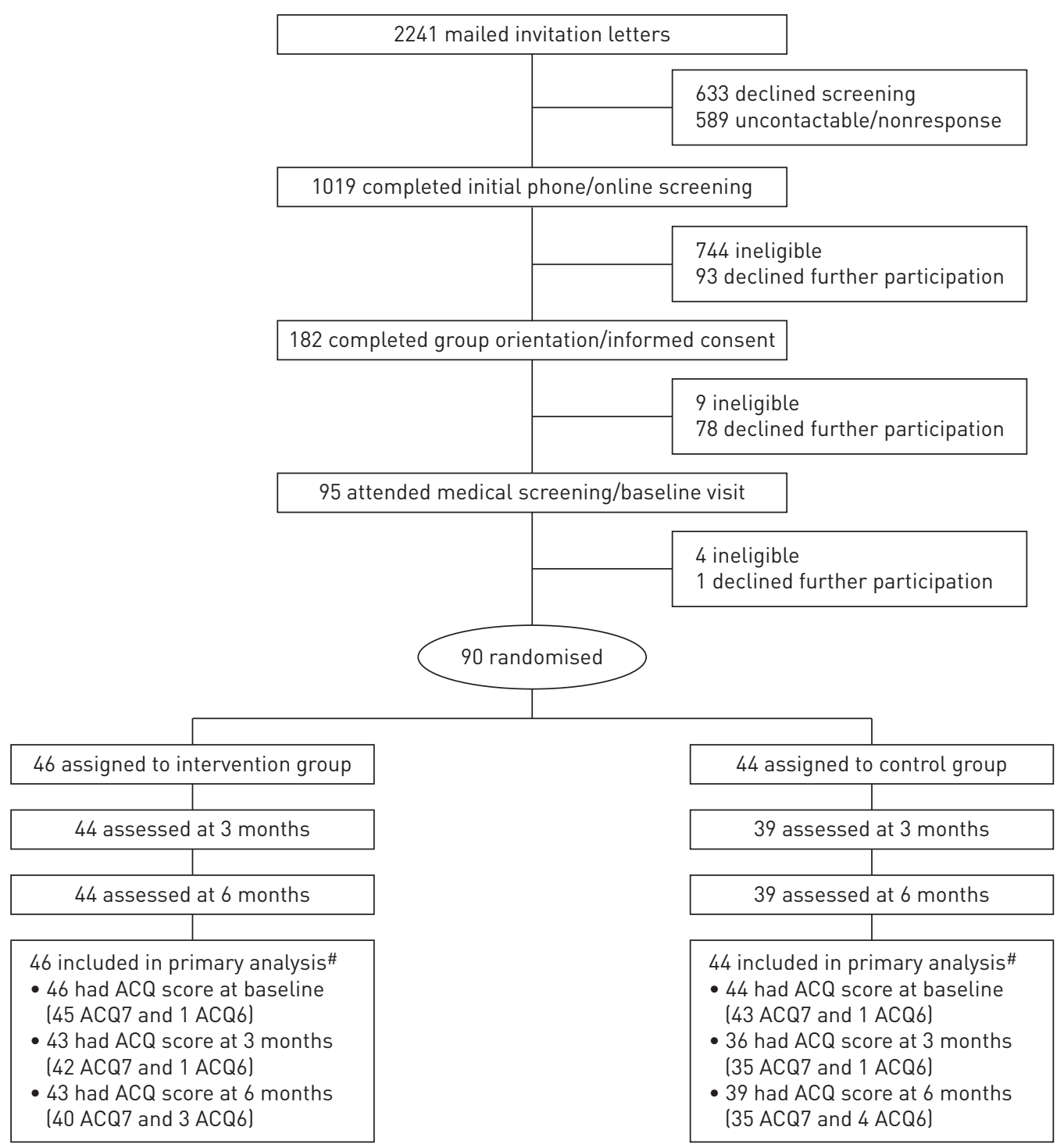

FIGURE 1 CONSORT chart. ACQ: Asthma Control Questionnaire. " : ACQ score was computed as the mean of the complete seven-item scores (ACQ7) or the six-item scores (ACQ6) if forced expiratory volume in $1 \mathrm{~s}$ was missing because spirometry was not performed or not usable per American Thoracic Society standards.

\section{Treatment arms}

\section{Intervention}

Intervention participants received a dietician-delivered behavioural intervention that promoted the DASH eating pattern as recommended in the 2010 US Dietary Guidelines [17]. The intervention curriculum included an intensive phase (three individual and eight group sessions, 60 min each, over 3 months) and a maintenance phase (monthly or more frequent phone consultations depending on participant needs, preferences and availability). The intervention was grounded in social cognitive theory [18]. Interventionists used proven behaviour change strategies (e.g. self-monitoring, action planning and problem solving) to help participants achieve and maintain primary DASH daily goals adapted to their individual caloric needs for weight maintenance: 1) seven to 12 servings of fruit and vegetables, 2) two to four servings of low-fat/fat-free dairy products, 3 ) total fat grams at $27 \%$ of estimated caloric needs, and 4$) \leqslant 2300 \mathrm{mg}$ of sodium. Exercise was not a component of the intervention. More intervention details are available in the protocol [11].

\section{Control}

Control participants received no intervention from the study. Regardless of group assignment, all participants continued to receive standard medical care from their providers, who were not informed of participants' treatment assignment. Each participant watched a Kaiser Permanente standard asthma self-management educational video $(10 \mathrm{~min})$ as part of the baseline assessment visit. 


\section{Outcome measures}

Of the 90 randomised participants, 83 (92\%) were assessed at 3 and 6 months (figure 1). All outcome assessors were trained to perform the measurements and interviews per standardised protocols and procedures.

The ACQ [10] integrates seven components of asthma control, including five patient-reported asthma symptoms, reported need for bronchodilators and measured FEV1; all are scored on a seven-point scale (0-6), with a higher score indicating worse asthma control. The ACQ score was computed as the mean of the seven-item scores (ACQ7) or the six-item scores (ACQ6) if FEV1 was missing because spirometry was not performed or the test was invalid. Both measures have been validated $[10,19]$ and we used the same approach in another published asthma trial [20]. There were 90 participants with ACQ scores (88 ACQ7 and two ACQ6) at baseline, 79 (77 and two) at 3 months, and 82 (75 and seven) at 6 months.

Participants also completed the 15-item Mini Asthma-specific Quality of Life Questionnaire (MiniAQLQ) [21]. A change of at least 0.5 points is regarded as clinically significant for both the ACQ and MiniAQLQ [19]. Outcome assessors performed spirometry according to the American Thoracic Society (ATS) standards [22], with ongoing quality monitoring. Two independent respiratory therapists rated all spirograms. A valid test required a minimum of two manoeuvres meeting the ATS acceptability and reproducibility criteria, from among which the best parameter values were chosen for FEV1 and forced vital capacity (FVC) separately and used in data analyses. Outcome assessors also followed published protocols to obtain weight and blood pressure measurements [23, 24].

For dietary intake, outcome assessors conducted multiple-pass 24-h diet recalls [25], which is the current gold standard for diet assessment, with participants over the phone using the Windows-based Nutrition Data System for Research (Nutrition Coordinating Centre, University of Minnesota, Minneapolis, MN, USA). These data were used to compute participants' DASH concordance index as a measure of overall diet quality [12]. Participants also completed fasting blood draws at the onsite clinical laboratory for assays of plasma glucose and lipid profile. Atopic status was determined by testing specific IgE antibodies to dust mite (Dermatophagoides pteronyssinus), animal dander (cat and dog), grasses (ragweed and timothy grass) and tree (juniper cedar) using ImmunoCAP Specific IgE (Phadia, Uppsala, Sweden).

Data abstracted from Kaiser Permanente of Northern California electronic health records included encounters with the healthcare system, diagnoses and pharmacy dispensing events during the 6 months before and after randomisation. Participants were interviewed at each follow-up visit about possible adverse events during the past 3 months and the study physician adjudicated the events per study safety protocol.

\section{Statistical analyses}

No participants were excluded after randomisation. Intention-to-treat analyses of between-treatment differences in primary and secondary outcomes tested for treatment-by-time interactions in repeated-measures mixed-effects linear (for continuous outcomes) or logistic models (for categorical outcomes). The fixed effects of each model consisted of the baseline value of the outcome of interest, randomisation balancing factors, treatment, time point and treatment-by-time interaction. The random effects accounted for repeated measures with a spatial power covariance structure which was selected based on the least Bayesian information criterion and for clustering of patients within primary care providers. Missing data were handled directly through maximum-likelihood estimation in mixed modelling. Least-square means (95\% confidence intervals) were obtained from the models. All analyses were conducted using SAS version 9.2 (SAS Institute, Cary, NC, USA).

As the primary goal of this pilot study was not hypothesis testing but estimation, we calculated the target sample size by controlling the length of the confidence interval, not by controlling the power of a statistical test at an appropriate alternative. We estimated that given a sample of 45 participants per arm with a projected $10 \%$ attrition at 6 months, the likelihood that the expected two-sided $95 \%$ confidence interval for an observed effect would have a standardised half-width no greater than 0.5 was $90 \%$ [26]. In other words, it was $90 \%$ or more likely that the estimated $95 \%$ confidence interval would not overlap the null if the observed effect was equivalent to a Cohen's $\mathrm{d}=0.5$ (medium effect) or larger. We specified $a$ priori various scenarios of the observed effect and confidence interval, and the corresponding decisions about whether a full-scale trial would be warranted (see the protocol [11]).

\section{Results}

\section{Baseline characteristics}

Participants were middle-aged, mostly female and never-smokers; they were racially/ethnically and socioeconomically diverse (table 1). At baseline, they had low DASH scores (mean \pm SD) of $2.3 \pm 1.3$ and

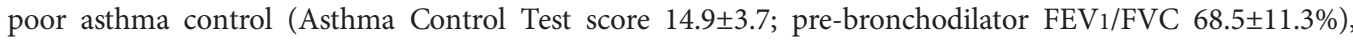


TABLE 1 Baseline characteristics of the study participants

\begin{tabular}{|c|c|c|c|}
\hline & All participants & Intervention & Control \\
\hline Subjects n & 90 & 46 & 44 \\
\hline Age years & $51.8 \pm 12.4$ & $52.2 \pm 11.9$ & $51.4 \pm 12.9$ \\
\hline Female & 67 & 72 & 61 \\
\hline \multicolumn{4}{|l|}{ Race/ethnicity } \\
\hline Non-Hispanic White & 43 & 46 & 41 \\
\hline Non-Hispanic Black & 11 & 13 & 9 \\
\hline Asian/Pacific Islander & 31 & 28 & 34 \\
\hline Hispanic/Latino & 14 & 13 & 16 \\
\hline \multicolumn{4}{|l|}{ Education } \\
\hline High school graduate or less & 9 & 2 & 16 \\
\hline Some college & 38 & 44 & 32 \\
\hline College graduate or above & 53 & 54 & 52 \\
\hline \multicolumn{4}{|l|}{ Employment status } \\
\hline Full-time & 59 & 67 & 50 \\
\hline Part-time & 10 & 9 & 11 \\
\hline Unemployed/retired/disabled & 31 & 24 & 39 \\
\hline \multicolumn{4}{|l|}{ Family annual income US\$ $n=87^{\#}$} \\
\hline$<35000$ & 15 & 13 & 17 \\
\hline $35000-<55000$ & 15 & 18 & 12 \\
\hline $55000-<75000$ & 18 & 11 & 26 \\
\hline $75000-<100000$ & 18 & 27 & 10 \\
\hline $100000-<125000$ & 13 & 16 & 10 \\
\hline $125000-<150000$ & 6 & 4 & 7 \\
\hline$\geqslant 150000$ & 15 & 11 & 19 \\
\hline DASH ${ }^{\text {ๆ }}$ score & $2.3 \pm 1.3$ & $2.4 \pm 1.4$ & $2.2 \pm 1.2$ \\
\hline \multicolumn{4}{|l|}{ Smoking status } \\
\hline Never-smoker & 74 & 81 & 68 \\
\hline Current smoker & 6 & 4 & 7 \\
\hline Ex-smoker & 20 & 15 & 25 \\
\hline Pack-years of current or ex-smokers $n=23^{\#}$ & $10.3 \pm 12.5$ & $9.4 \pm 10.3$ & $10.9 \pm 14.0$ \\
\hline $\begin{array}{l}\text { Asthma onset age before } 12 \text { years by } \\
\text { self-report }\end{array}$ & 29 & 26 & 32 \\
\hline Atopy $\mathrm{n}=\mathbf{7 7 ^ { \# , + }}$ & 74 & 72 & 76 \\
\hline ACT & $14.9 \pm 3.7$ & $15.2 \pm 3.8$ & $14.6 \pm 3.6$ \\
\hline ACQ & $1.3 \pm 0.8$ & $1.2 \pm 0.7$ & $1.4 \pm 0.8$ \\
\hline FEV 1 L $\mathbf{n}=88^{\#}$ & $2.6 \pm 0.8$ & $2.5 \pm 0.9$ & $2.7 \pm 0.7$ \\
\hline FVC L $n=88^{\#}$ & $3.8 \pm 1.0$ & $3.6 \pm 1.0$ & $3.9 \pm 1.0$ \\
\hline $\mathrm{FEV} 1 / \mathrm{FVC} n=88^{\#}$ & $68.5 \pm 11.3$ & $67.8 \pm 11.1$ & $69.3 \pm 11.5$ \\
\hline
\end{tabular}

Data are presented as mean \pm SD or $\%$, unless otherwise stated. DASH: Dietary Approaches to Stop Hypertension (range 0-9 with higher scores indicating better DASH concordance); ACT: Asthma Control Test (range 5-25 with higher scores indicating better asthma control); ACQ: Asthma Control Questionnaire (range 0-6 with higher scores indicating worse asthma control); FEV1: forced expiratory volume in $1 \mathrm{~s}$; FVC: forced vital capacity. " : denominator was <90 randomised participants because of missing data. ๆ: DASH scores were calculated based on combining nine nutrient targets (i.e. total fat, saturated fat, protein, cholesterol, fibre, magnesium, calcium, sodium and potassium). The intermediate target of each nutrient was half-way between the DASH target and population mean (based on the National Health and Nutrition Examination Surveys 2007-2008, latest data available at the inception of this study). For a nutrient, participants reaching the DASH target were assigned one point, those reaching the intermediate target were assigned a half-point, and those not meeting the intermediate target were given 0 points. The DASH score was the sum of points for all nine nutrients [12]. ${ }^{+}$: atopy was defined as any tested aeroallergen having a value $\geqslant 0.35$ based on ImmunoCAP Specific IgE testing. The types of allergies for which specific IgE tests were performed included dust mite (Dermatophagoides pteronyssinus), two types of animal dander (cat and dog), two grasses (ragweed and timothy grass) and tree (juniper cedar).

and ACQ averaged $1.3 \pm 0.8 ; 29 \%$ had asthma onset before 12 years of age by self-report and $74 \%$ were atopic according to a positive result $\left(\geqslant 0.35 \mathrm{kU} \cdot \mathrm{L}^{-1}\right)$ for any of the six allergen-specific IgEs tested.

Overall diet quality and daily goal attainment

Mixed-model-based estimates showed that intervention participants had a significant net-of-control increase of $0.8(95 \%$ CI $0.2,1.5)$ in DASH score and $1.9(0.8,3.0)$ daily servings of fruit and vegetables at 
6 months (table 2), which were also observed at 3 months (online supplementary table E2). Figure 2 shows the complete fitted distributions and sorted individual values of change in DASH scores. The Cohen's d estimate based on unadjusted mean \pm SD changes in DASH scores over 6 months of the two groups was 0.5 , suggesting medium intervention effect (figure 3a). In addition, at 6 months intervention participants showed a net mean increase in low-fat/fat-free dairy servings, and net mean decreases in fat and sodium intake, although the $95 \%$ confidence intervals included the null. The percentage of intervention participants meeting the minimum daily DASH goals was $33 \%$ versus $8 \%$ of controls for seven servings of fruit and vegetables, $49 \%$ versus $53 \%$ for total fat grams at $27 \%$ of individualised caloric needs, $23 \%$ versus $15 \%$ for two servings of low-fat/fat-free dairy products, and $58 \%$ versus $40 \%$ for $\leqslant 2300 \mathrm{mg}$ of sodium. Compared with the control group, the intervention group's log-transformed odds ratios of reaching the minimum goal were greater for fruit and vegetable servings and sodium intake, although only the latter reached statistically significance (figure 4 ).

\section{Asthma control, asthma-specific functional status and lung function}

At 6 months, intervention participants had a net improvement of -0.2 (95\% CI -0.5, 0.1) in ACQ score, and consistently favourable changes in MiniAQLQ overall and subdomain scores (figure 3a). The unadjusted Cohen's d estimates ranged from 0.28 to 0.48 for these asthma outcomes, indicating small-to-medium intervention effects. Significantly higher percentages of intervention participants than controls achieved clinically important improvements of at least 0.5 in ACQ scores (28\% versus 9\%, $\mathrm{p}=0.04$ ) and overall MiniAQLQ scores (55\% versus 16\%, $\mathrm{p}=0.004$ ) (figure $3 \mathrm{~b}$ ). ACQ and MiniAQLQ results were unaffected by further adjustment for asthma long-term controller (i.e. beclomethasone canister equivalents,

TABLE 2 Estimated mean changes from baseline to 6 months in DASH score, asthma outcomes, weight and blood pressure in the intention-to-treat population

\begin{tabular}{|c|c|c|c|c|}
\hline & \multicolumn{4}{|c|}{ Change from baseline to 6 months } \\
\hline & Baseline & Intervention & Control & Difference \\
\hline \multicolumn{5}{|l|}{ Intervention goal measure } \\
\hline DASH score & $2.3 \pm 1.3$ & $0.6 \pm 0.3$ & $-0.3 \pm 0.3$ & $0.8(0.2,1.5)$ \\
\hline Fruits and vegetables servings per day & $4.4 \pm 2.4$ & $1.6 \pm 0.5$ & $-0.3 \pm 0.5$ & $1.9(0.8,3.0)$ \\
\hline Fat g.day ${ }^{-1}$ & $66.3 \pm 40.7$ & $-5.3 \pm 4.8$ & $-4.7 \pm 4.7$ & $-0.5(-11.6,10.5)$ \\
\hline Low fat/fat-free dairy servings per day ${ }^{\#}$ & $1.0 \pm 0.9$ & $0.2 \pm 0.2$ & $-0.1 \pm 0.2$ & $0.3(-0.1,0.7)$ \\
\hline Sodium $\mathrm{mg} \cdot \mathrm{day}^{-1}$ & $2718 \pm 1278$ & $-117 \pm 197$ & $-58 \pm 192$ & $-58(-529,413)$ \\
\hline \multicolumn{5}{|l|}{ Asthma control } \\
\hline $\mathrm{ACQ}$ & $1.3 \pm 0.8$ & $-0.2 \pm 0.1$ & $-0.0 \pm 0.1$ & $-0.2(-0.5,0.1)$ \\
\hline \multicolumn{5}{|l|}{ Asthma-specific functional status } \\
\hline MiniAQLQ, overall & $5.2 \pm 1.1$ & $0.7 \pm 0.2$ & $0.2 \pm 0.2$ & $0.4(0.0,0.8)$ \\
\hline MiniAQLQ, symptoms & $5.2 \pm 1.3$ & $0.6 \pm 0.2$ & $0.1 \pm 0.2$ & $0.5(0.0,0.9)$ \\
\hline MiniAQLQ, environment & $4.6 \pm 1.6$ & $0.7 \pm 0.2$ & $0.3 \pm 0.2$ & $0.4(-0.1,1.0)$ \\
\hline MiniAQLQ, emotions & $4.9 \pm 1.5$ & $0.7 \pm 0.2$ & $0.3 \pm 0.2$ & $0.4(-0.2,0.9)$ \\
\hline MiniAQLQ, activities & $6.0 \pm 1.0$ & $0.6 \pm 0.2$ & $0.2 \pm 0.2$ & $0.3(-0.0,0.7)$ \\
\hline \multicolumn{5}{|l|}{ Spirometry lung function test } \\
\hline FEV1 L & $2.6 \pm 0.8$ & $-0.0 \pm 0.0$ & $-0.0 \pm 0.0$ & $0.0(-0.1,0.1)$ \\
\hline FVC L & $3.8 \pm 1.0$ & $0.0 \pm 0.1$ & $0.1 \pm 0.1$ & $-0.0(-0.2,0.1)$ \\
\hline $\mathrm{FEV}_{1} / \mathrm{FVC} \%$ & $68.5 \pm 11.3$ & $-0.4 \pm 1.1$ & $-1.6 \pm 1.0$ & $1.2(-1.3,3.7)$ \\
\hline \multicolumn{5}{|l|}{ Weight and blood pressure } \\
\hline Weight kg & $76.5 \pm 15.9$ & $-1.2 \pm 0.7$ & $-1.1 \pm 0.7$ & $-0.1(-1.8,1.5)$ \\
\hline Body mass index $\mathrm{kg} \cdot \mathrm{m}^{-2}$ & $27.9 \pm 4.8$ & $-0.5 \pm 0.2$ & $-0.4 \pm 0.2$ & $-0.1(-0.7,0.4)$ \\
\hline Systolic blood pressure $\mathrm{mmHg}$ & $117.4 \pm 14.4$ & $-5.5 \pm 1.7$ & $-3.6 \pm 1.6$ & $-2.0(-5.9,2.0)$ \\
\hline Diastolic blood pressure $\mathrm{mmHg}$ & $74.9 \pm 8.9$ & $-1.5 \pm 1.1$ & $0.7 \pm 1.1$ & $-2.2(-4.7,0.4)$ \\
\hline \multicolumn{5}{|c|}{$\begin{array}{l}\text { Data are presented as mean } \pm \text { SD (Baseline), covariate-adjusted, mixed-model-based mean } \pm \text { SE (Intervention } \\
\text { and Control) or covariate-adjusted, mixed-model-based mean }(95 \% \text { CI) (Difference). DASH: Dietary } \\
\text { Approaches to Stop Hypertension (range 0-9 with higher scores indicating better DASH concordance); ACQ: } \\
\text { Asthma Control Questionnaire (range 0-6 with higher scores indicating worse asthma control); MiniAQLQ: } \\
\text { Mini Asthma Quality of Life Questionnaire; FEV1: forced expiratory volume in } 1 \mathrm{~s} \text {; FVC: forced vital capacity. } \\
\text { \#: servings were calculated using the Nutrition Data System for Research per the } 2000 \text { Dietary Guidelines } \\
\text { for Americans (fruit: one serving= } 1 \text { medium apple, banana, orange or pear, } 0.5 \text { cups of chopped, cooked or } \\
\text { canned fruit, } 0.25 \text { cups dried fruit or } 4 \mathrm{fl} \text {. oz of fruit juice; vegetables: one serving=1 cup of raw leafy } \\
\text { vegetables, } 0.5 \text { cups of other cooked or raw vegetables or } 4 \mathrm{fl} \text {. oz of vegetable juice; dairy: one serving=1 } \\
\text { cup of milk or yogurt, } 1.5 \mathrm{oz} \text { of natural cheese or } 2 \text { oz of processed cheese). }\end{array}$} \\
\hline
\end{tabular}



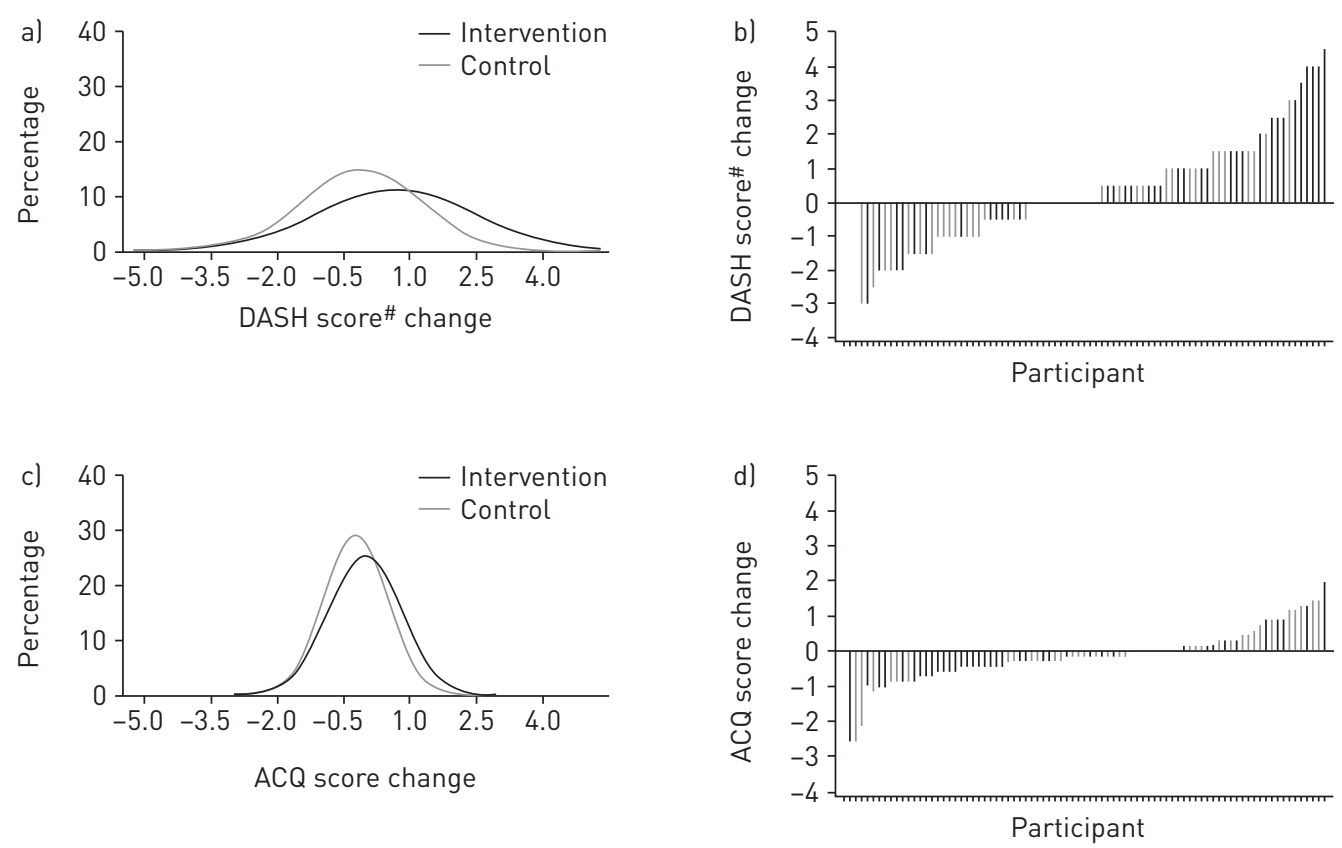

FIGURE 2 Fitted distributions and sorted values of changes in a, b) Dietary Approaches to Stop Hypertension (DASH) and $c, d$ ) Asthma Control Questionnaire (ACQ) scores at 6 months. "\#: DASH scores were calculated based on combining nine nutrient targets (i.e. total fat, saturated fat, protein, cholesterol, fibre, magnesium, calcium, sodium and potassium). The intermediate target of each nutrient was half-way between the DASH target and population mean (based on the National Health and Nutrition Examination Surveys 2007-2008, latest data available at the inception of this study). For a nutrient, participants reaching the DASH target were assigned 1 point, those reaching the intermediate target were assigned 0.5 points and those not meeting the intermediate target were given 0 points. The DASH score was the sum of points for all nine nutrients [12].

a measure of the anti-inflammatory equipotency [27]) or/and short-acting $\beta$-agonist (SABA) medication use (i.e. albuterol canister equivalents, a measure of the bronchodilator equipotency) (data not shown).

Spirometric parameters (FEV1, FVC and FEV1/FVC), weight and BMI were stable in both groups (table 2). Participants had normal mean blood pressure, fasting plasma glucose and fasting plasma lipids at baseline, with nonsignificant improvements in the intervention relative to the control group (table 2 and online supplementary table E3).

\section{Asthma-related medications and healthcare encounters}

Based on pharmacy dispensing records during the 6 months before randomisation, $21 \%$ of the 90 participants did not acquire any asthma controller medications, $72 \%$ acquired SABAs, $20 \%$ had asthma exacerbations requiring systemic corticosteroids, $1 \%$ had asthma-related hospitalisations and $11 \%$ had asthma-related emergency department visits. The corresponding numbers were $23 \%, 62 \%, 13 \%, 2 \%$ and $9 \%$, respectively, during the 6 months after randomisation. The mean \pm sD beclomethasone canister equivalents of the total acquired long-term controller medications (excluding theophylline and maintenance systemic corticosteroids used for $>15$ days) were $8.3 \pm 8.9$ and $7.9 \pm 9.0$ in 6 months pre- and post-randomisation, respectively. The mean \pm SD albuterol canister equivalents of the total acquired SABAs were $1.9 \pm 2.2$ and $1.7 \pm 1.9$ over the same time periods. Intervention participants showed greater mean increases in long-term controller use and in outpatient visits, whereas they had greater mean decreases in SABA use and in asthma exacerbations requiring systemic corticosteroids; however, the confidence intervals were wide and all included the null (table 3).

\section{Adverse events}

Two hospitalisations occurred during the 6-month trial; neither was study-related and one was unexpected. This event involved a 41-year-old female intervention participant who presented to the emergency department with an asthma exacerbation, was treated with systemic corticosteroids and epinephrine, and then intubated and admitted to the intensive care unit for 2 days because of severe respiratory distress and acidosis. The patient was discharged with a corrected, stable respiratory status, and with prescription for a 5 -day course of prednisone and azithromycin. The other hospitalisation occurred in a control participant with a history of persistent cough, acid reflux and abdominal pain who underwent laparoscopic hiatal hernia repair surgery. There were no deaths. 


$\begin{array}{lc}\text { a) } & \text { Baseline }^{\#} \\ \text { DASH score } & 2.3 \pm 1.3 \\ \text { ACQ } & 1.3 \pm 0.8 \\ \text { MiniAQLQ, overall } & 5.2 \pm 1.1 \\ \text { MiniAQLQ, symptoms } & 5.2 \pm 1.3 \\ \text { MiniAQLQ, environment } & 4.6 \pm 1.6 \\ \text { MiniAQLQ, emotions } & 4.9 \pm 1.5 \\ \text { MiniAQLQ, activities } & 6.0 \pm 1.0\end{array}$

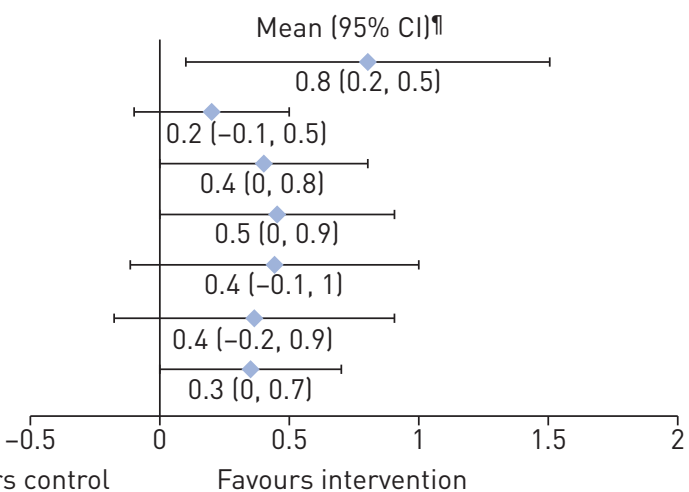

Cohen's d
0.50
0.28
0.46
0.42
0.32
0.36
0.41

Favours control Favours intervention

b) Intervention Control

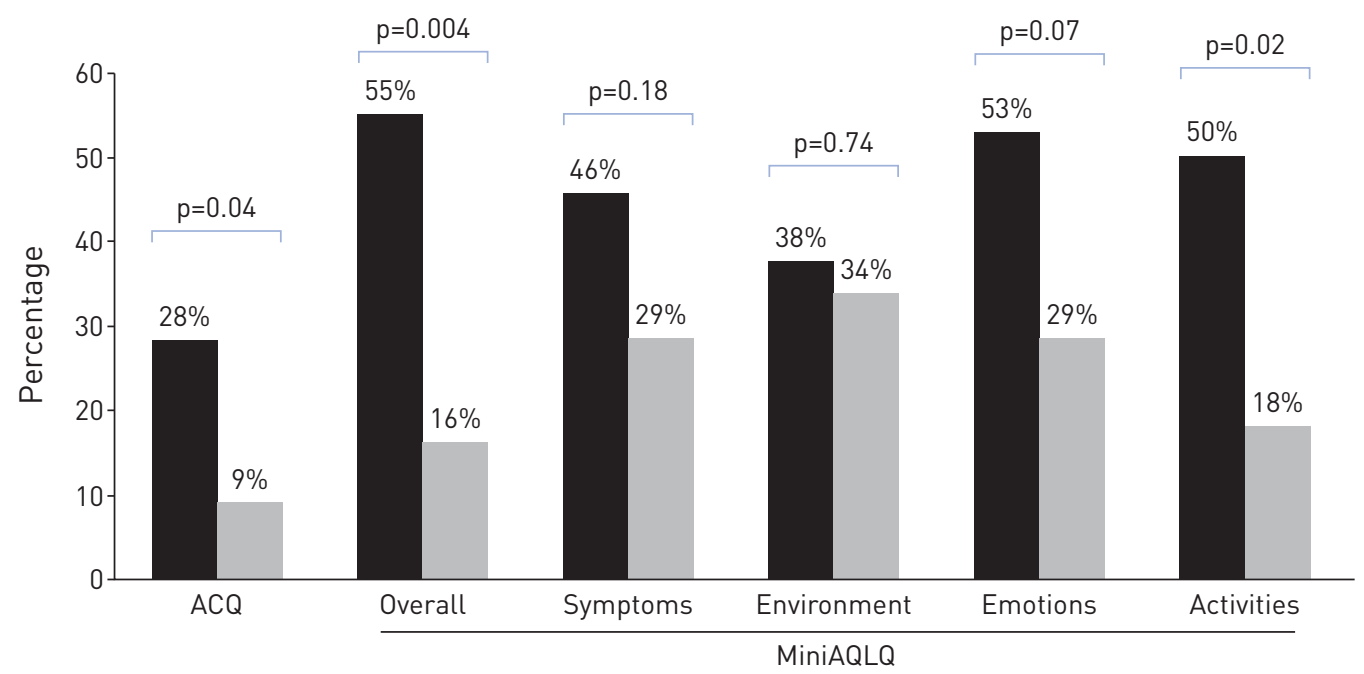

FIGURE 3 a) Baseline DASH, ACQ and MiniAQLQ scores, and their net-of-control improvements at 6 months. b) Percentage of participants meeting minimal clinically significant improvement $(\geqslant 0.5)$ in ACQ and MiniAQLQ at 6 months. DASH: Dietary Approaches to Stop Hypertension; ACQ: Asthma Control Questionnaire; MiniAQLQ: Mini Asthma-specific Quality of Life Questionnaire. \#: mean $\pm \mathrm{SD}$; ": covariate-adjusted, mixed-model-based mean $(95 \% \mathrm{Cl}) ;{ }^{+}$: calculated as unadjusted between-group mean difference in change from baseline to 6 months divided by pooled standard deviation.

\section{Discussion}

This was the first RCT of a behavioural intervention promoting the DASH eating pattern to examine the effects of a whole diet on asthma control. Compared with the control group, we found that the intervention led to significantly improved overall diet quality, and consistently favourable improvements in asthma control and related functional status as assessed by the ACQ and MiniAQLQ. A Cohen's d of 0.28 for the

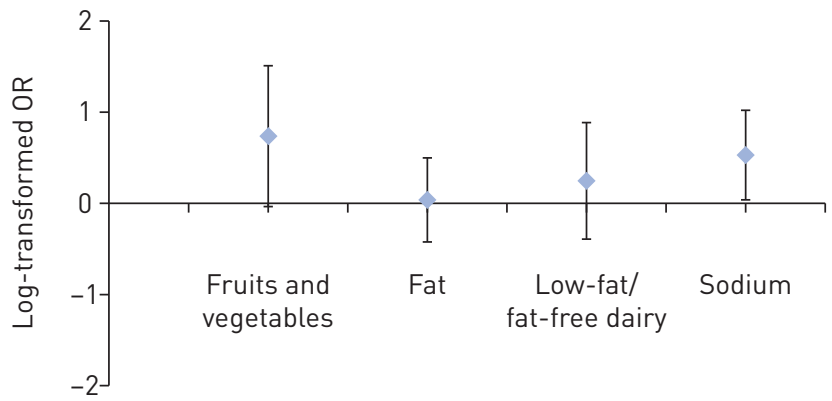

FIGURE 4 Log-transformed odds ratios of intervention participants relative to controls meeting minimum Dietary Approaches to Stop Hypertension intervention goals at 6 months. Bars indicate $95 \%$ confidence interval. 
TABLE 3 Changes in asthma-related medication use and healthcare encounters ${ }^{\#}$

\begin{tabular}{ccccc}
6 months before randomisation & & \multicolumn{2}{c}{6 months after randomisation } & Difference \\
$\begin{array}{c}\text { All } \\
\text { participants }\end{array}$ & Intervention Control & $\begin{array}{c}\text { All } \\
\text { participants }\end{array}$ & Intervention Control &
\end{tabular}

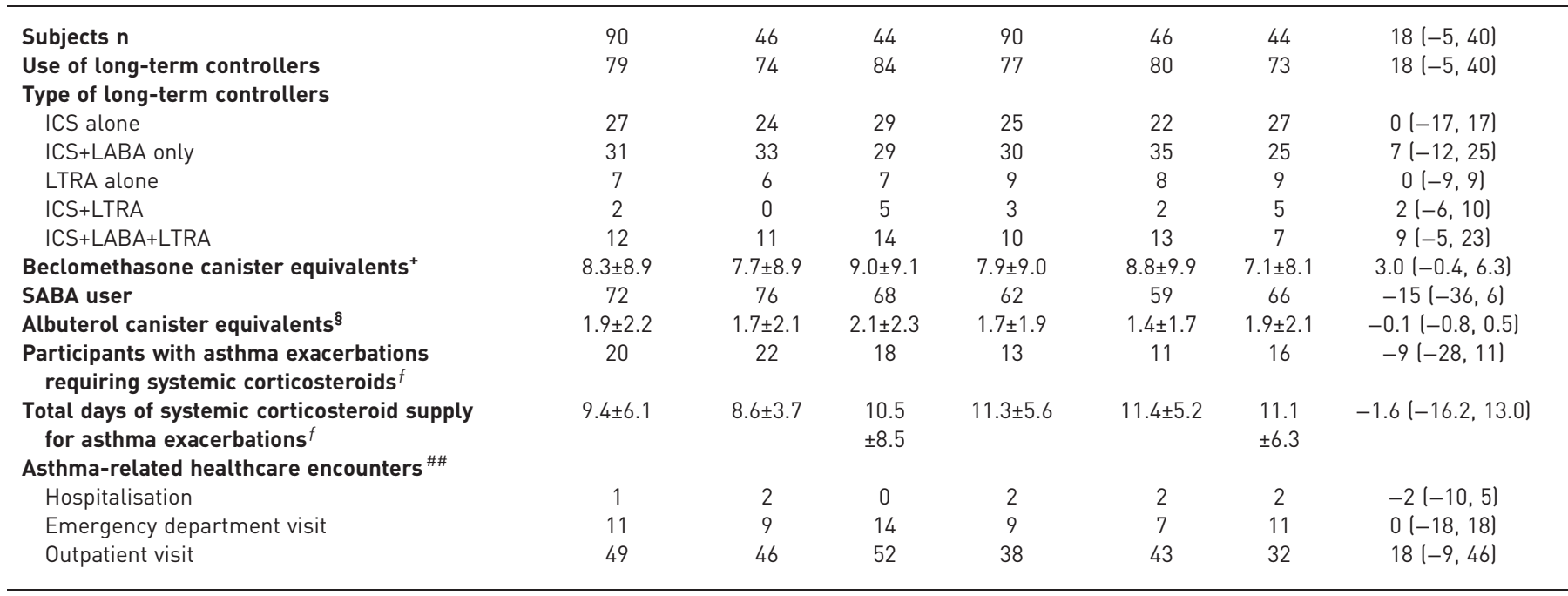

Data are presented as mean \pm SD or $\%$, unless otherwise stated. ICS: inhaled corticosteroid; LABA: long-acting, inhaled $\beta_{2}$-agonist; LTRA: leukotriene modifier; SABA: short-acting $\beta$-agonist. \#: extracted healthcare utilisation data from electronic health records included all visits by service type (emergency department, hospital inpatient or outpatient department) to Kaiser Permanente in Northern California and its contracted facilities, and pharmacy dispensing records; ": covariate-adjusted, mixed-model-based mean $195 \% \mathrm{Cl}$ ) for between-treatment differences in change from 6 months before to 6 months after randomisation; ${ }^{+}$: estimated total number of canisters of beclomethasone to which an asthma controller medication dispensed for a patient in each 6-month period was equivalent in terms of its anti-inflammatory effectiveness (each dispensing of a controller medication (excluding theophylline and systemic corticosteroids used for $>15$ days), in any form or quantity, was assigned a weight representing its equivalent in fractional or multiple canisters of beclomethasone $80 \mathrm{mg}$ [27]; weighted values were summed for each patient to obtain a cumulative measure of controller medication dose dispensed during the 6 months before and after randomisation separately); ${ }^{\S}$ : estimated total number of canisters of albuterol to which the amount of SABA dispensed in each 6-month period was equivalent in terms of its bronchodilator effectiveness leach SABA medication, in any type or delivery mode, was assigned a weight representing its equivalent in canister of albuterol [27]; weighted values were summed for each patient to obtain a cumulative measure of dose dispensed during the 6 months before and after randomisation separately); ${ }^{\prime}$ : defined as systemic corticosteroids (by tablet, suspension or injection) dispensed for 3-15 days of use; \#\#: asthma-related healthcare encounters were visits with International Classification of Diseases, ninth revision (Clinical Modification) code 493 as a primary or secondary diagnosis.

ACQ change qualifies as a probably positive finding according to the various scenarios pre-specified in the study protocol about the possible intervention effect [11]. Greater Cohen's d values were observed for improvements in overall and subdomain MiniAQLQ scores. The net-of-control mean improvement in the MiniAQLQ symptoms subdomain was 0.5 among intervention participants, which is considered clinically significant [19]. Furthermore, significantly higher percentages of intervention participants than controls achieved clinically significant improvements in ACQ and overall MiniAQLQ scores. Even though the confidence intervals for asthma medication use and asthma exacerbations (as well as cardiovascular risk factors) were wide, and almost all included the null, they changed consistently in favour of the intervention.

The available literature on the relationship between diet and asthma manifestations has focussed on individual nutrients and selected foods or food groups. A few intervention studies among adults with asthma investigated the relationship between individual nutrients and asthma manifestations using dietary supplements (e.g. vitamin C, vitamin E, fish oil/ $\omega$-3 fatty acid and selenium) or dietary modification (e.g. sodium reduction); however, the results have been largely disappointing [28-32]. Some epidemiologic studies have shown a protective effect of fruit, vegetable and fish intake on asthma manifestations in adults $[5,33,34]$. Other epidemiologic studies examined the relationship between dietary patterns and asthma outcomes, including asthma control, asthma-related quality of life, asthma symptoms and lung function [9]. Emerging data from mechanistic studies suggest oxidative stress and inflammatory response may explain the role of healthy eating in asthma symptomology and controls $[35,36]$.

In a two-stage RCT, Wood et al. [37] randomly assigned adults with objectively confirmed asthma to a high-antioxidant diet (five servings of vegetables and two servings of fruit daily; $\mathrm{n}=46$ ) or a low-antioxidant diet (two or less servings of vegetables and one serving of fruit daily; $\mathrm{n}=91$ ) for 14 days, at 
which point remaining participants of the latter group were further randomised to receive placebo $(n=33)$ or tomato extract ( $45 \mathrm{mg}$ lycopene daily; $\mathrm{n}=31$ ) and all those in the high-antioxidant diet group $(\mathrm{n}=40)$ received placebo [37]. The intervention continued until week 14 or until an exacerbation occurred; 79 (58\%) of the initial 137 participants attended final visits. The authors concluded that a high-antioxidant diet led to improved FEV1 and FVC, and a lower risk of asthma exacerbation, compared with a low-antioxidant diet, associated with evidence of reduced systemic inflammation, but that the antioxidant supplement had no effect on inflammation or clinical outcomes compared with placebo. Despite its shortcomings (e.g. short duration, high attrition and unclear level of blinding), the trial provided the first direct evidence that a whole-food approach rich in fruit and vegetables could be more important than isolated antioxidant supplementation in the management of asthma. As noted earlier, another small $(\mathrm{n}=38)$ and short duration (12 weeks) RCT concluded that promoting a Mediterranean diet was feasible, and worth examining for its clinical benefit, in adults with symptomatic asthma [8].

The current study contributes importantly to this very limited dietary pattern-asthma RCT literature by being the first RCT of a DASH-promoting behavioural intervention. The methodological limitations (e.g. small sample size, short intervention and follow-up duration) are reflective of a pilot study. Importantly, the intervention under study significantly improved DASH scores, an index of overall diet quality, mostly attributable to increased fruit and vegetable intake and reduced sodium intake over the 6-month period. The magnitudes of improvement in DASH scores [38] and in individual daily dietary goal achievements (e.g. fruit, vegetables and dairy) [39, 40] are comparable with similar dietary interventions in different populations and settings. Study participants reported an average of 4.4 servings of fruit and vegetables at baseline, which may be higher than usual intake of the general US adult population, possibly due to greater access to fruit and vegetables in California [41, 42]. Generally, US adults who have lower fruit and vegetable intake may have a higher likelihood of observing an improvement in overall diet quality with the intervention. This pilot trial showed, for the first time, that a DASH-promoting behavioural intervention significantly improved diet quality with promising clinical benefits for better asthma control and functional status among adults with uncontrolled asthma. The intervention proved to be safe, with no evidence of increased adverse effects on asthma medication use, exacerbations or acute healthcare utilisation (hospitalisations and emergency visits). These results provide good evidence that full-scale RCTs to more definitively examine the efficacy of this intervention and any modifications that may enhance its effectiveness are warranted.

\section{Acknowledgements}

We are indebted to the following individuals for their instrumental contributions to the conduct of the study: Veronica Luna, Andrea Blonstein, Elizabeth Jameiro and Nancy Wittels (Palo Alto Medical Foundation Research Institute, Palo Alto, CA, USA); Ranna Modir, Jennifer Waldrop and Jodi Thirtyacre (Kaiser Permanente of Northern California, San Francisco, CA, USA). We wish to thank the DASH Data and Safety Monitoring Board members, William Haskell, Ware G. Kuschner and Manisha Desai (Stanford University, Stanford, CA, USA), and extend special thanks to the DASH participants and their families who made this study possible. We also would like to acknowledge Unni C. Nygaard (Stanford University, Stanford, CA, USA) for her comments on the manuscript. The study was conducted while J. Ma was a faculty member at the Palo Alto Medical Foundation Research Institute.

\section{References}

1 National Center for Health Statistics. National Health Interview Survey, 2012. www.cdc.gov/asthma/nhis/2012/ table3-1.htm Date last accessed: March 7, 2014.

2 Global Initiative for Asthma (GINA). Global Strategy for Asthma Management and Prevention, 2007. http://www. ginasthma.org/documents/4 Date last accessed: October 6, 2015.

3 Allen S, Britton JR, Leonardi-Bee JA. Association between antioxidant vitamins and asthma outcome measures: systematic review and meta-analysis. Thorax 2009; 64: 610-619.

4 Barros R, Moreira A, Fonseca J, et al. Dietary intake of alpha-linolenic acid and low ratio of n-6:n-3 PUFA are associated with decreased exhaled NO and improved asthma control. Br J Nutr 2011; 106: 441-450.

5 Iikura M, Yi S, Ichimura Y, et al. Effect of lifestyle on asthma control in Japanese patients: importance of periodical exercise and raw vegetable diet. PLoS One 2013; 8: e68290.

6 Barros R, Moreira A, Fonseca J, et al. Adherence to the Mediterranean diet and fresh fruit intake are associated with improved asthma control. Allergy 2008; 63: 917-923.

7 Guo CH, Liu PJ, Lin KP, et al. Nutritional supplement therapy improves oxidative stress, immune response, pulmonary function, and quality of life in allergic asthma patients: an open-label pilot study. Altern Med Rev 2012; 17: 42-56.

8 Sexton $\mathrm{P}$, Black $\mathrm{P}$, Metcalf $\mathrm{P}$, et al. Influence of mediterranean diet on asthma symptoms, lung function, and systemic inflammation: a randomized controlled trial. J Asthma 2013; 50: 75-81.

9 Lv N, Xiao L, Ma J. Dietary pattern and asthma: a systematic review and meta-analysis. J Asthma Allergy 2014; 7: $105-121$.

10 Juniper EF, O’Byrne PM, Guyatt GH, et al. Development and validation of a questionnaire to measure asthma control. Eur Respir J 1999; 14: 902-907.

11 Ma J, Strub P, Lavori PW, et al. DASH for asthma: a pilot study of the DASH diet in not-well-controlled adult asthma. Contemp Clin Trials 2013; 35: 55-67. 

dietary accordance, NHANES 1988-1994 and 1999-2004. Arch Intern Med 2008; 168: 308-314.

13 Nathan RA, Sorkness CA, Kosinski M, et al. Development of the asthma control test: a survey for asse asthma control. J Allergy Clin Immunol 2004; 113: 59-65.

14 Ma J, Yank V, Xiao L, et al. Translating the Diabetes Prevention Program lifestyle intervention for weight loss into primary care: a randomized trial. JAMA Intern Med 2013; 173: 113-121.

15 Pocock SJ, Simon R. Sequential treatment assignment with balancing for prognostic factors in the controlled clinical trial. Biometrics 1975; 31: 103-115.

16 Efron B. Forcing sequential experiment to be balanced. Biometrika 1971; 58: 403-417.

17 US Department of Health and Human Services and US Department of Agriculture. 2010 Dietary Guidelines for Americans, 2011. www.cnpp.usda.gov/dietaryguidelines.htm Date last accessed: August 23, 2011.

18 Bandura A. Social foundations of thought and action: a social cognitive theory. Englewood Cliffs, Prentice Hall, 1986.

19 Juniper EF, Svensson K, Mork AC, et al. Measurement properties and interpretation of three shortened versions of the asthma control questionnaire. Respir Med 2005; 99: 553-558.

20 Ma J, Strub P, Xiao L, et al. Behavioral weight loss and physical activity intervention in obese adults with asthma. A randomized trial. Ann Am Thorac Soc 2015; 12: 1-11.

21 Juniper EF, Guyatt GH, Cox FM, et al. Development and validation of the Mini Asthma Quality of Life Questionnaire. Eur Respir J 1999; 14: 32-38.

22 Miller MR, Hankinson J, Brusasco V, et al. Standardisation of spirometry. Eur Respir J 2005; 26: 319-338.

23 Hamilton CM, Strader LC, Pratt JG, et al. The PhenX Toolkit: get the most from your measures. Am J Epidemiol 2011; 174: 253-260.

24 Pickering TG, Hall JE, Appel LJ, et al. Recommendations for blood pressure measurement in humans and experimental animals: Part 1: blood pressure measurement in humans: a statement for professionals from the Subcommittee of Professional and Public Education of the American Heart Association Council on High Blood Pressure Research. Hypertension 2005; 45: 142-161.

25 Conway JM, Ingwersen LA, Vinyard BT, et al. Effectiveness of the US Department of Agriculture 5-step multiple-pass method in assessing food intake in obese and nonobese women. Am J Clin Nutr 2003; 77: 1171-1178.

26 Bristol DR. Sample sizes for constructing confidence intervals and testing hypotheses. Stat Med 1989; 8: 803-811.

Schatz M, Nakahiro R, Crawford W, et al. Asthma quality-of-care markers using administrative data. Chest 2005; 128: 1968-1973.

28 Pearson PJ, Lewis SA, Britton J, et al. Vitamin E supplements in asthma: a parallel group randomised placebo controlled trial. Thorax 2004; 59: 652-656.

29 Fogarty A, Lewis SA, Scrivener SL, et al. Oral magnesium and vitamin C supplements in asthma: a parallel group randomized placebo-controlled trial. Clin Exp Allergy 2003; 33: 1355-1359.

30 Shaheen SO, Newson RB, Rayman MP, et al. Randomised, double blind, placebo-controlled trial of selenium supplementation in adult asthma. Thorax 2007; 62: 483-490.

31 Pogson ZE, Antoniak MD, Pacey SJ, et al. Does a low sodium diet improve asthma control? A randomized controlled trial. Am J Respir Crit Care Med 2008; 178: 132-138.

32 Moreira A, Moreira P, Delgado L, et al. Pilot study of the effects of n-3 polyunsaturated fatty acids on exhaled nitric oxide in patients with stable asthma. J Investig Allergol Clin Immunol 2007; 17: 309-313.

33 Patel BD, Welch AA, Bingham SA, et al. Dietary antioxidants and asthma in adults. Thorax 2006; 61: $388-393$.

34 Laerum BN, Wentzel-Larsen T, Gulsvik A, et al. Relationship of fish and cod oil intake with adult asthma. Clin Exp Allergy 2007; 37: 1616-1623.

35 Sienra-Monge JJ, Ramirez-Aguilar M, Moreno-Macias H, et al. Antioxidant supplementation and nasal inflammatory responses among young asthmatics exposed to high levels of ozone. Clin Exp Immunol 2004; 138: $317-322$.

36 Mickleborough TD, Lindley MR, Ray S. Dietary salt, airway inflammation, and diffusion capacity in exercise-induced asthma. Med Sci Sports Exerc 2005; 37: 904-914.

37 Wood LG, Garg ML, Smart JM, et al. Manipulating antioxidant intake in asthma: a randomized controlled trial. Am J Clin Nutr 2012; 96: 534-543.

38 Kwan MW, Wong MC, Wang HH, et al. Compliance with the Dietary Approaches to Stop Hypertension (DASH) diet: a systematic review. PLoS One 2013; 8: e78412.

39 Pomerleau J, Lock K, Knai C, et al. Interventions designed to increase adult fruit and vegetable intake can be effective: a systematic review of the literature. J Nutr 2005; 135: 2486-2495.

40 Appel LJ, Champagne CM, Harsha DW, et al. Effects of comprehensive lifestyle modification on blood pressure control: main results of the PREMIER clinical trial. JAMA 2003; 289: 2083-2093.

41 United States Centers for Disease Control and Prevention. State indicator report on fruits and vegetables, 2013. www.cdc.gov/nutrition/downloads/State-Indicator-Report-Fruits-Vegetables-2013.pdf Date last accessed: June 1, 2015.

42 Sugerman S, Foerster SB, Gregson J, et al. California adults increase fruit and vegetable consumption from 1997-2007. J Nutr Educ Behav 2011; 43: S96-S103. 